Engineering, Technology and Techniques

Vol.59: e16161076, January-December 2016 http://dx.doi.org/10.1590/1678-4324-2016161076 ISSN 1678-4324 Online Edition

BRAZILIAN ARCHIVES OF BIOLOGY AND TECHNOLOGY

AN INTERNATIONAL JOURNAL

\title{
HRL-Local Infinite Triangular Array Languages
}

\author{
V.Devi Rajaselvi ${ }^{1 *}$, T.Kalyani ${ }^{2}$, D.G.Thomas ${ }^{3}$. \\ ${ }^{1}$ Research Scholar,Sathyabama University, Chennai,India; ${ }^{2}$ Department of Mathematics,St.Joseph's Institute of \\ Technology, Chennai,India; ${ }^{3}$ Department of Mathematics,Madras Christian College, Chennai,India.
}

\begin{abstract}
A new subclass of infinite triangular arrays called hrl-local infinite triangular arrays is introduced. We introduce infinite triangular domino systems to recognize the infinite triangular picture language. Also we introduce strictly domino testable $\omega \omega$-triangular array languages.

Key words: hrl-local infinite triangular arrays, infinite triangular domino systems, strictly dommo testable $\omega \omega$ -
\end{abstract} triangular array languages.

\footnotetext{
*Author for correspondence: gsramcivil34@gmail.com
} 


\section{INTRODUCTION}

Infinite triangular pictures are the digitized images which occur in the triangular grid of two dimensional plane. Infinite triangular picture $p$ is a triangular array of elements of the terminal alphabet. It is useful to introduce the notation $\Sigma_{\mathrm{T}}^{\infty}$ for the set of all infinite triangular pictures over the same alphabet $\Sigma$. Infinite triangular picture has infinite number of rows and infinite number of right slanting lines and infinite number of left slanting lines. The size $|p|$ of a picture $p$ is specified by the pair $\left(|\mathrm{p}|_{\text {row }},|\mathrm{p}|_{\text {rsline }},|\mathrm{p}|_{\text {sline }}\right)$ of its number of rows and right slanting lines and left slanting lines. A pixel $\mathrm{p}(\mathrm{i}, \mathrm{j}, \mathrm{k}), 1 \leq \mathrm{i} \leq|\mathrm{p}|_{\text {row }}, 1 \leq \mathrm{j} \leq|\mathrm{p}|_{\text {rsline }}, 1 \leq \mathrm{j} \leq|\mathrm{p}|_{\text {sline }}$ is the element at position $(\mathrm{i}, \mathrm{j}, \mathrm{k})$ in the triangular array P. Conventionally the indices grow from bottom to top for the rows and from right to left for left slanting lines and from left to right for right slanting lines.

For convenience we usually consider the bordered version of picture $\mathrm{p}$ obtained by surrounding the picture with the special boundary symbol \# which is assumed not to be in the alphabet. In ${ }^{2}$ domino recognizability of triangular picture languages and hrl-domino systems are defined. Also in ${ }^{2}$ we define the overlapping of isotriangular pictures.

In ${ }^{1}$ we learn about recognizable infinite array languages. In ${ }^{6}$ we study the recognizability of infinite arrays and in ${ }^{3}$ we define the recognizability of infinite triangular pictures.

In this paper we consider another formalism hrl-domino system to recognize infinite triangular picture languages.

\section{INFINITE TRIANGULAR PICTURE AND DOMINO SYSTEMS}

Infinite triangular picture has infinite number of rows and infinite number of right slanting and left slanting lines. The set of all infinite triangular arrays over $\Sigma$ is denoted by $\Sigma_{\text {IT }}^{\omega \infty}$.

In ${ }^{4,5}$ hv-local picture languages are defined, where the square tiles of side 2 are replaced by dominoes that correspond to two kinds of tiles (i) horizontal dominoes of size $(1,2)$ and (ii) vertical dominoes of size $(2,1)$. As in ${ }^{2}$ here we consider dominoes of the following types

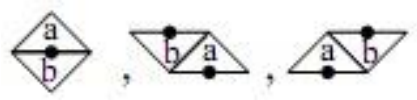

Definition 1. $\mathrm{L} \subseteq \sum_{\mathrm{IT}}^{\infty}$ is hrl-local if there exists a finite set $\Delta$ of dominoes over $\Sigma \cup 1 \nRightarrow \square$ $\mathrm{L}(\Delta)$.

Definition 2. An infinite triangular domino system is a 4-tuple ITD $=(\Sigma, \Gamma, \Delta, \pi)$ where $\Sigma$ and $\Gamma$ are two finite alphabets, $\Delta$ is a finite set of dominoes over the alphabet $\Gamma \cup \leadsto$ and $\pi: \Gamma \rightarrow \Sigma$ is a projection.

An infinite triangular domino system recognizes an infinite triangular picture language $\mathrm{L}$ over the alphabet $\Sigma$ and is defined as $\mathrm{L}=\pi\left(\mathrm{L}^{\prime}\right)$ where $\mathrm{L}^{\prime}=\mathrm{L}(\Delta)$ is the hrl-local infinite triangular picture language over $\Gamma$. The family of infinite triangular picture languages recognizable by infinite triangular domino systems is denoted by L(ITDS). 
Proposition 1. If $\mathrm{L} \subseteq \sum_{\mathrm{IT}}^{\infty}$ is a hrl-local infinite triangular picture language then $\mathrm{L}$ is a local infinite triangular picture language. That is $\mathrm{L}($ ITDS $) \subseteq \mathrm{L}($ ITTS).

Proof. Let $\mathrm{L} \subseteq \Sigma_{\mathrm{IT}}^{\infty}$ is a hrl-local infinite triangular picture language. Then $\mathrm{L}=$ $\mathrm{L}(\Delta)$ where $\Delta$ is a finite set of dominoes. We will construct a finite set $\theta$ of $\omega \omega$ triangular arrays of size 2 and show that $\mathrm{L}=\mathrm{L}(\theta)$.

We now show that $L^{\prime}=L$. Let $p \in L^{\prime}$. Then by definition $B_{1,2}(\hat{p}) \in \theta$. This implies that

$\mathrm{B}_{2,1}(\hat{\mathrm{p}}) \subseteq \mathrm{B}_{2,1}\left(\mathrm{~B}_{2,1}(\hat{\mathrm{p}})\right) \subseteq \mathrm{B}_{2,1}(\theta) \subseteq \Delta$. Hence $\mathrm{p} \in \mathrm{L}$.

Conversely let $\mathrm{p} \in \mathrm{L}$ and $\mathrm{q} \in \mathrm{B}_{1,2}(\hat{\mathrm{p}})$. Then $\mathrm{B}_{2,1}(\mathrm{q}) \subseteq \mathrm{B}_{2,1}(\hat{\mathrm{p}}) \subseteq \Delta$. Therefore $\mathrm{q} \in \theta$ and

Hence $\mathrm{L}=\mathrm{L}^{\prime}$.

$\mathrm{p} \in$

$\mathrm{L}^{\prime}$.

Lemma 1. Let $\mathrm{L}$ be a local $\omega \omega$-triangular picture language over an alphabet $\Sigma$. Then there exists an hrl local language $L^{\prime}$ over an alphabet and a mapping $\pi: \Gamma \rightarrow$ $\Sigma$ such that $\mathrm{L}=\pi\left(\mathrm{L}^{\prime}\right)$.

Proof. Let $\mathrm{L}=\mathrm{L}(\theta)$ where $\theta$ is a finite set of $\omega \omega$-triangular arrays over $\Sigma \cup$

Let $\mathrm{S}_{1}, \mathrm{~S}_{2}, \mathrm{~S}_{\mathbf{3}} \subseteq \Sigma_{\mathrm{T}}^{* *}$ be three strings languages over $\Sigma$. The hrl-combination of $\mathrm{S}_{\mathbf{1}}$, $\mathrm{S}_{2}, \mathrm{~S}_{3}$ is an triangular array language $\mathrm{L}_{\mathrm{T}}=\mathrm{S}_{\mathbf{1}} \oplus \mathrm{S}_{\mathbf{2}} \oplus \mathrm{S}_{3} \subseteq \Sigma_{\mathrm{T}}^{* *}$ such that $\mathrm{p} \in \mathrm{L}$ if and only if the strings corresponding to the hrl overlapping of $p$ belong to $S_{1}, S_{2}$ and to $S_{3}$ respectively. Also we have to prove that the class of all hrl-local triangular array languages is the hrl combination of the class of all local string languages.

Theorem 1. hrl-TLOC $=$ TLOC $\oplus$ TLOC $\oplus$ TLOC.

Proof. Let $\mathrm{L} \in \operatorname{hrl}$-TLOC. Then $\mathrm{L}=\mathrm{L}_{\mathrm{T}}^{* *}(\Delta)$, for a finite set of dominoes over $\Sigma \cup$ $\{\#\}$. Let

$$
\begin{aligned}
& I_{1}=\{a: \stackrel{\mathrm{a}}{\#} \in \in \Delta \\
& \left.b: \frac{\hat{A}}{\mathrm{~b}} \in \Delta\right\} \text {, } \\
& \left.C_{1}=\left\{a b: \Delta \frac{\Delta}{b}\right\} \in \Delta\right\}, \\
& J_{1}=\{a: \stackrel{\mathrm{a}}{\#} \in \Delta \\
& a: \frac{\vec{B}}{\mathrm{~b}} \in \Delta \text {, } \\
& l_{2}=\{a: \triangle \mathbb{A} \in \Delta\}, \\
& C_{2}=\{a b:\langle\Delta \overrightarrow{\mathrm{a} b} \in \Delta\} \text {, } \\
& J_{2}=\{a: \Delta \mathbb{A} \sharp \in \Delta\} . \\
& l_{3}=\{a: \mathbb{\# / a} \in \Delta\} \text {, } \\
& c_{3}=\{a b: \text { b/a } \in \Delta\} \text {, } \\
& J_{3}=\{a: b / \nexists \Delta\} \text {. }
\end{aligned}
$$

Braz. Arch. Biol. Technol. v.59: e16161076 Jan/Dec 2016 Spec Iss 2 
Let $\mathrm{L}_{1}, \mathrm{~L}_{2}$ and $\mathrm{L}_{3}$ be the hrl-local string languages generated by the local sys-tems $\left(\mathrm{I}_{1}, \mathrm{C}_{1}, \mathrm{~J}_{1}\right),\left(\mathrm{I}_{2}, \mathrm{C}_{2}, \mathrm{~J}_{2}\right)$ and $\left(\mathrm{I}_{3}, \mathrm{C}_{3}, \mathrm{~J}_{3}\right)$ respectively. Then $\mathrm{L}=\mathrm{L}_{1} \oplus \mathrm{L}_{2} \oplus \mathrm{L}_{3} \in \mathrm{TLOC}$ $\oplus \mathrm{TLOC} \oplus \mathrm{TLOC}$.

Conversely let $\mathrm{L} \in \mathrm{TLOC} \oplus \mathrm{TLOC} \oplus \mathrm{TLOC}$. Let $\mathrm{L}=\mathrm{L}_{1} \oplus \mathrm{L}_{2} \oplus \mathrm{L}_{3}$ where $\mathrm{L}_{1}, \mathrm{~L}_{2}$ and $\mathrm{L}_{3}$ are the local string languages generated by the local systems $\left(\mathrm{I}_{1}, \mathrm{C}_{1}, \mathrm{~J}_{1}\right),\left(\mathrm{I}_{2}\right.$, $\left.\mathrm{C}_{2}, \mathrm{~J}_{2}\right)$ and $\left(\mathrm{I}_{3}, \mathrm{C}_{3}, \mathrm{~J}_{3}\right)$ respectively. Let

$$
\begin{aligned}
& \Delta_{1}=\left\{\stackrel{\mathrm{a}}{\#}: a \in I_{1}, \frac{\#}{\mathrm{~b}}: b \in I_{1}\right\}, \\
& \Delta_{2}=\left\{\hat{\mathrm{b}}: a b \in C_{1}\right\}, \\
& \Delta_{3}=\left\{\stackrel{\text { a }}{\#}: a \in J_{1}, \frac{\#}{\mathrm{~b}}: b \in J_{1}\right\}, \\
& \Delta_{4}=\left\{\not \mathrm{b} /: a \in I_{2}\right\}, \\
& \Delta_{5}=\left\{\Delta \mathrm{a} /: a \in C_{2}\right\} \text {, }
\end{aligned}
$$

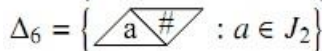

$$
\begin{aligned}
& \Delta_{7}=\left\{\# / \mathrm{a}: a \in I_{3}\right\}, \\
& \Delta_{8}=\left\{\mathrm{b} / \mathrm{a}: a \in C_{3}\right\} \text {, } \\
& \Delta_{9}=\left\{\mathrm{b} / \#: a \in J_{3}\right\}
\end{aligned}
$$

Let $\Delta=\Delta_{1} \cup \Delta_{2} \cup \Delta_{3} \cup \Delta_{4} \cup \Delta_{5} \cup \Delta_{5} \cup \Delta_{6} \cup \Delta_{7} \cup \Delta_{8} \cup \Delta_{9}$. Then $\mathrm{L}=\mathrm{L}_{\mathrm{T}}^{* *}(\Delta)$ and therefore $\mathrm{L} \in$ hrl-TLOC.

\section{Example 1.}

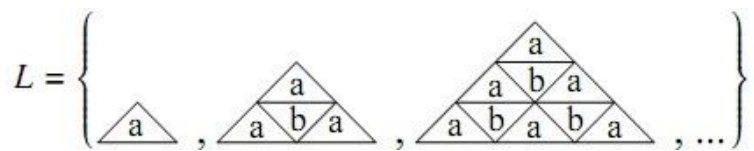

is the hrl-local language which is represented by the following set of dominoes.

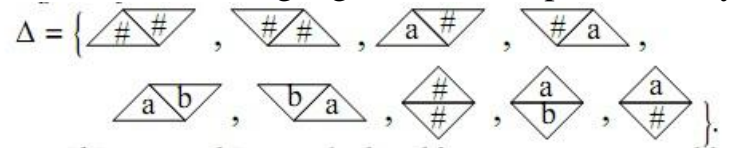

If $\mathrm{L}_{1}, \mathrm{~L}_{2}$ and $\mathrm{L}_{3}$ are the local languages generated by the local systems $\mathrm{S}_{1}=\left\{\mathrm{I}_{1}, \mathrm{C}_{1}\right.$, $\left.\mathrm{J}_{1}\right\}$ and $\mathrm{S}_{2}=\left\{\mathrm{I}_{2}, \mathrm{C}_{2}, \mathrm{~J}_{2}\right\}$ respectively where

$I_{1}=\{a\}, C_{1}=\{a b\}, J_{1}=\{a\}$,

$\mathrm{I}_{2}=\varphi, \mathrm{C}_{2}=\{\mathrm{ab}\}, \mathrm{J}_{2}=\{\mathrm{a}\}$,

$\mathrm{I}_{3}=\{\mathrm{a}\}, \mathrm{C}_{3}=\{\mathrm{ab}\}, \mathrm{J}_{3}=\varphi$,

$\mathrm{L}_{1}=\left\{(\mathrm{ab})^{*}\right\}, \mathrm{L}_{2}=\left\{(\mathrm{ab})^{*} \mathrm{a}\right\}$. Then $\mathrm{L}=\mathrm{L}_{1} \oplus \mathrm{L}_{2} \oplus \mathrm{L}_{3}$.

Definition 3. Let $\mathrm{L} \subseteq \sum_{\mathrm{IT}}^{\infty}$, we denote by $\mathrm{B}_{\mathrm{h}, \mathrm{k}, \mathrm{l}}(\mathrm{p})$, domino testable, if there exists a finite set of dominoes of size $(1,1, l+1),(1, \mathrm{k}+1,1)$ and $(\mathrm{h}+1,1,1)$ such that $\mathrm{L}=\left\{\mathrm{p} \in \Sigma_{\mathrm{TT}}^{\omega 0}: \mathrm{B}_{\mathbf{1 , 1 , l + 1}}(\hat{\mathrm{p}}) \cup \mathrm{B}_{\mathbf{1 , k + 1 , 1}}(\hat{\mathrm{p}}) \cup \mathrm{B}_{\mathrm{h}+\mathbf{1}, \mathbf{1}, \mathbf{1}}(\hat{\mathrm{p}}) \subseteq \Delta\right\}$

and we write $\mathrm{L}=\mathrm{L}_{\mathrm{T}}^{\omega_{0}}(\Delta)$. The family of all strictly $(\mathrm{h}, \mathrm{k}, l)$-domino testable $\omega \omega$ triangular array languages is denoted by $\mathrm{SDTT}_{\mathrm{h}, \mathrm{k} \downarrow}^{\omega \omega}$. Let $\mathrm{L} \subseteq \Sigma_{\mathrm{IT}}^{\omega \infty}$ is strictly domino testable, if $\mathrm{L} \subseteq \mathrm{SDTT}_{\mathrm{IT}}^{\omega}$, for some $\mathrm{h}, \mathrm{k}>0$. The family of all strictly domino testable $\omega \omega$-triangular languages is denoted by $\mathrm{SDTT}^{\omega \omega}$. Here we notice that 
HRL-Local Infinite Triangular Array Languages

$$
\begin{aligned}
& \operatorname{SDTT}_{\mathrm{h}, k, l}^{\omega \omega} \subseteq \operatorname{SDTT}_{\mathrm{h}+1, k, l}^{\omega \omega} \\
& \operatorname{SDTT}_{\mathrm{h}, k, l}^{\omega \omega} \subseteq \operatorname{SDTT}_{\mathrm{h}, \mathrm{k}+1,}^{\omega \omega} \\
& \text { and } \\
& \operatorname{SDTT}_{\mathrm{h}, \mathrm{k}, l}^{\omega \omega} \subseteq \operatorname{SDTT}_{\mathrm{h}, \mathrm{k}, 1+1}^{\omega \omega}
\end{aligned}
$$

Example 2. Let $\Sigma=\{a, b\}$ and

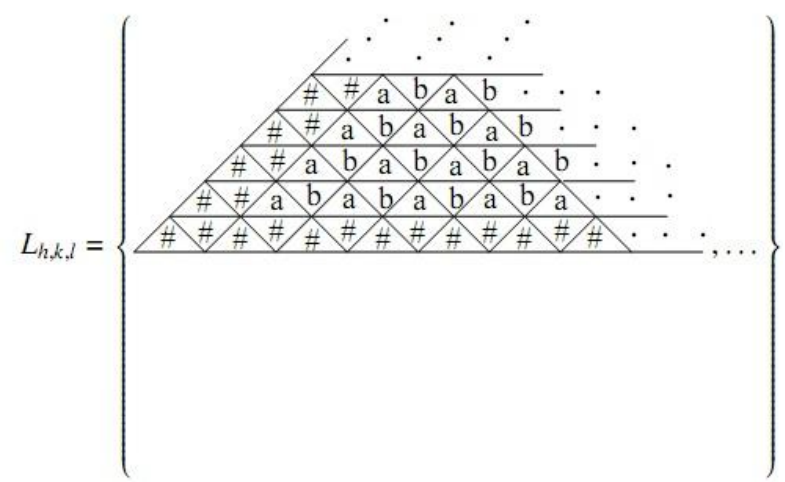

In $\mathrm{L}_{\mathrm{h}, \mathrm{k}, l}$ the entries in the $(\mathrm{i}, \mathrm{j}, \mathrm{k})^{\text {th }}$ position for $\mathrm{i}>\mathrm{h}$ and $\mathrm{j}>\mathrm{k}$ and $\mathrm{k}>l$ are $\mathrm{a}$ and $\mathrm{b}$ and other entries are $a_{1}$ and $b_{1}$ then

$$
\begin{aligned}
& \mathrm{L}_{\mathrm{h}+1, \mathrm{k}, \downarrow} \in \mathrm{SDTT}_{\mathrm{h}+1, \mathrm{k}, \downarrow}^{\omega \omega} \backslash \mathrm{SDTT}_{\mathrm{h}, \mathrm{k}, l}^{\omega \omega} \text {, } \\
& \mathrm{L}_{\mathrm{h}, \mathrm{k}+1, \downarrow} \in \mathrm{SDTT}_{\mathrm{h}, \mathrm{k}+1, \downarrow}^{\omega \omega} \backslash \mathrm{SDTT}_{\mathrm{h}, \mathrm{k}, \downarrow}^{\omega \omega} \text {, } \\
& \mathrm{L}_{\mathrm{h}, \mathrm{k}, l+1} \in \mathrm{SDTT}_{\mathrm{h}, \mathrm{k},+1}^{\omega \omega} \backslash \mathrm{SDTT}_{\mathrm{h}, \mathrm{k}, l}^{\omega \omega} \text {. }
\end{aligned}
$$

Thus we have

$$
\begin{array}{ccc}
\vdots & \vdots & \vdots \\
\cup & \cup & \cup \\
\operatorname{SDTT}_{2,1,1}^{\omega \omega} & \sim \operatorname{SDTT}_{2,2,1}^{\omega \omega} & \sim \operatorname{SDTT}_{2,3,1}^{\omega \omega} \subset \cdots \\
\cup & \cup \\
\operatorname{SDTT}_{1,1,1}^{\omega \omega} & \cup \operatorname{SDTT}_{1,2,1}^{\omega \omega} & \sim \operatorname{SDTT}_{1,3,1}^{\omega \omega} \subset \cdots
\end{array}
$$

Theorem 2.

$$
\begin{aligned}
& \operatorname{SDTT}_{\mathrm{h}, \mathrm{k},}^{\omega \omega}=\mathrm{SFLT}_{\mathrm{k}+1}^{\omega} \oplus \mathrm{SFLT}_{\mathrm{h}+1}^{\omega} \oplus \mathrm{SFLT}_{l+1}^{\omega} \quad \text { and } \\
& \mathrm{SDTT}^{\omega \omega}=\mathrm{SFLT}^{\omega} \oplus \mathrm{SFLT}^{\omega} \oplus \mathrm{SFLT}^{\omega} .
\end{aligned}
$$

Proof. Let 


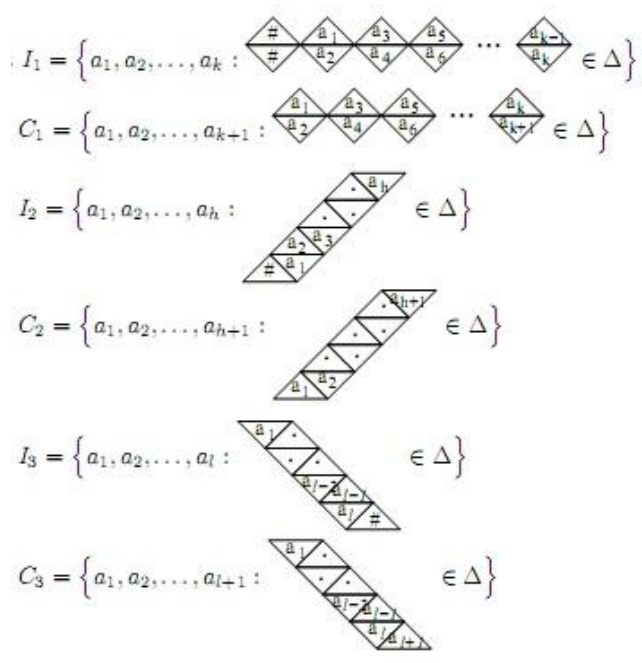

Let $\mathrm{L}_{1}, \mathrm{~L}_{2}$ and $\mathrm{L}_{3}$ be the members of $\mathrm{SFLT}_{\mathrm{k}+1}^{\omega}, \mathrm{SFLT}_{\mathrm{h}+1}^{\omega}$ and $\mathrm{SFLT}_{l+1}^{\omega}$ generated by local systems $\left(\mathrm{I}_{1}, \mathrm{C}_{1}\right),\left(\mathrm{I}_{2}, \mathrm{C}_{2}\right)$ and $\left(\mathrm{I}_{3}, \mathrm{C}_{3}\right)$ respectively. Let

$$
\begin{aligned}
& \Delta_{1}=\left\{a_{1}, a_{2}, \ldots, a_{k}: \Delta \sqrt{a_{1} / a_{2}} \cdot a_{k} \in I_{1}\right\} \\
& \Delta_{2}=\left\{a_{1}, a_{2}, \ldots, a_{k+1}: \Delta a_{1} 2 / a_{3} \cup \cdot a_{k} \rightarrow C_{1}\right\} \\
& \Delta_{3}=\left\{a_{1}, a_{2}, \ldots, a_{h_{1}}:\right. \\
& \Delta_{4}=\left\{a_{1}, a_{2}, \ldots, a_{h+1}\right. \\
& \Delta_{5}=\left\{a_{1}, a_{2}, \ldots, a_{l+1}:\right.
\end{aligned}
$$

Let $\Delta=\Delta_{1} \cup \Delta_{2} \cup \Delta_{3} \cup \Delta_{4} \cup \Delta_{5} \cup \Delta_{6}$. Then $\mathrm{L}_{1} \oplus \mathrm{L}_{2} \oplus \mathrm{L}_{3}=\mathrm{L}_{\mathrm{T}}^{\omega}(\Delta)$ where $\mathrm{L}_{1} \oplus \mathrm{L}_{2}$ $\oplus \mathrm{L}_{3} \in \mathrm{SDTT}_{\mathrm{h}, \mathrm{k}, \downarrow}^{\omega \omega}$. Thus we proved.

\section{CONCLUSION}

In this paper the notion of recognizability of infinite triangular pictures by a new formalism called hrl-domino systems have been investigated. Learning algorithm and automata characterization of hrl-local $\omega \omega$-triangular array languages will be studied. The learning of infinite triangular pictures and unary infinite triangular picture languages and their complexity deserve to be studied further.

\section{REFERENCES}

1. Dare VR, Subramanian KG, Thomas DG, Siromoney R. Infinite arrays and recognizabiltiy. Int. J. Pattern Recognition and Artificial Intelligence. 2000; 14: 525-536. 2. Devi Rajaselvi V, Kalyani T, Thomas DG. Domino recognizability of triangular picture languages. International Journal of Computer Applications. 2012; 57(15). 
HRL-Local Infinite Triangular Array Languages

3. Devi Rajaselvi V, Kalyani T, Thomas DG. Infinite triangular arrays and recognisability. Applied Mathematical Sciences. 2014; 8(106): 5269-5275.

4. Gnanasekaran S, Dare VR. Infinite arrays and domino systems. Electronic Notes in Discrete Mathematics. 2003; 12.

5. Gnanasekaran S, Dare VR. hv-local and prefix picture languages. Proc. National Conference on Recent Trends in Computational Mathematics. 2004.

6. Gnanasekaran S, Dare VR. On recognizable infinite array languages. Lecture Notes in Computer Science. 2004; 3322; 204-213.

Received: February 03, 2016; Accepted: July 14, 2016

Braz. Arch. Biol. Technol. v.59: e16161076 Jan/Dec 2016 Spec Iss 2 